\title{
ARTICLE OPEN \\ Combination of anti-PD-1 antibody with P-GEMOX as a potentially effective immunochemotherapy for advanced natural killer/T cell lymphoma
}

Jun Cai ${ }^{1,2}$, Panpan $\mathrm{Liu}^{1,2}$, Huiqiang Huang ${ }^{1,2}$, Yajun $\mathrm{Li}^{3,4}$, Shuyun Ma ${ }^{1,2}$, Hui Zhou ${ }^{3,4}$, Xiaopeng Tian ${ }^{1,2}$, Yuchen Zhang ${ }^{1,2}$, Yan Gao ${ }^{1,2}$, Yi Xia ${ }^{1,2}$, Xuanye Zhang ${ }^{1,2}$, Hang Yang ${ }^{1,2}$, Lirong $\mathrm{Li}^{1,2}$ and Qingqing Cai ${ }^{1,2}$

\begin{abstract}
Advanced natural killer/T cell lymphoma (NKTL) has demonstrated poor prognosis with currently available therapies. Here, we report the efficacy of anti-programmed death 1 (PD-1) antibody with the P-GEMOX (pegaspargase, gemcitabine, and oxaliplatin) regimen in advanced NKTL. Nine patients underwent six 21-day cycles of anti-PD-1 antibody (day 1 ), pegaspargase $2000 \mathrm{U} / \mathrm{m}^{2}$ (day 1), gemcitabine $1 \mathrm{~g} / \mathrm{m}^{2}$ (days 1 and 8 ) and oxaliplatin $130 \mathrm{mg} / \mathrm{m}^{2}$ (day 1), followed by anti-PD-1 antibody maintenance every 3 weeks. Programmed death-ligand 1 (PD-L1) expression and genetic alterations were determined in paraffin-embedded pretreatment tissue samples using immunohistochemistry and next-generation sequencing (NGS) analysis. Responses were assessed using ${ }^{18}$ F-fluorodeoxyglucose positron emission tomography $\left({ }^{18} \mathrm{FDG}\right.$-PET) and computed tomography or magnetic resonance imaging. Eight patients exhibited significant responses, comprising of seven complete remissions and one partial remission (overall response rate: $88.9 \%$ ). After a median follow-up of 10.6 months, $6 / 9$ patients $(66.7 \%)$ remained in complete remission. The most common grade $3 / 4$ adverse events were anemia (33.3\%), neutropenia (33.3\%), and thrombocytopenia (33.3\%); all of which were manageable and resolved. Immunochemotherapy produced a high response rate in patients with positive PD-L1 expression (5/6, 83.3\%). NGS analysis suggested that STAT3/JAK3/PD-L1 alterations and ARID1A mutation were associated with immunochemotherapy efficacy. Mutation in DDX3X and alteration in epigenetic modifiers of KMT2D, TET2, and BCORL1 might indicate a poor response to immunochemotherapy. In conclusion, the anti-PD-1 antibody plus P-GEMOX regimen demonstrated promising efficacy in advanced NKTL. PD-L1 expression combined with specific genetic alterations could be used as potential biomarkers to predict therapeutic responses to immunochemotherapy.
\end{abstract}

Signal Transduction and Targeted Therapy (2020)5:289

; https://doi.org/10.1038/s41392-020-00331-3

\section{INTRODUCTION}

Natural killer/T-cell lymphoma (NKTL) is a well-characterized subtype of peripheral T-cell lymphoma that is more common in East Asia and Latin America. ${ }^{1,2}$ More than two-thirds of NKTL patients have stage I or II diseases in the upper aerodigestive tract at the time of diagnosis., ${ }^{3,4}$ The prognosis of this subgroup of patients has been significantly improved with the use of concurrent chemoradiotherapy or sequential chemoradiotherapy with non-anthracycline chemotherapy..$^{5-7}$ In contrast to localized NKTL where front-line therapy may be associated with long-term remission in over $60 \%$ of patients, the optimal treatment for advanced NKTL remains a major challenge as $70-80 \%$ of the patients experience disease progression or death within 5 years of diagnosis. $^{8-11}$

Asparaginase and pegaspargase are key components of chemotherapeutic regimens for advanced NKTL. ${ }^{12-14}$ However, treatment-related adverse events (AEs) still remain a significant challenge. Several studies have suggested that pegaspargase, gemcitabine, and oxaliplatin (P-GEMOX) might have high efficacy while exhibiting better tolerability, and is recommended as the first-line treatment. In a retrospective study of 10 years' real-world clinical experience in the treatment of NKTL from China, the P-GEMOX regimen provided an overall response rate (ORR) of $71.7 \%$ in advanced NKTL, with a 2-year progression-free survival (PFS) rate of $33.8 \%$, and a 2-year overall survival (OS) rate of $44.5 \% .{ }^{15}$ In addition, a recent prospective study by Huang et al. ${ }^{16}$ showed that P-GEMOX plus thalidomide regimen had an ORR of $87.1 \%$ and a complete response (CR) rate of $56.3 \%$ in advanced or relapsed/refractory $(r / r)$ NKTL, with a 3 -year PFS and OS of $47.0 \%$ and $44.3 \%$, respectively. However, it is also important to note that $\sim 70 \%$ of patients would still relapse despite first-line chemotherapy. Currently, the long-term survival rate of patients with advanced NKTL is still low. Thus, new drugs and effective therapeutic approaches are urgently needed.

NKTL has a high frequency of programmed death-ligand 1 (PDL1) expression, which is upregulated by the Epstein-Barr virus

\footnotetext{
${ }^{1}$ State Key Laboratory of Oncology in South China, Collaborative Innovation Center of Cancer Medicine, Sun Yat-sen University Cancer Center, Guangzhou 510060, P.R. China;

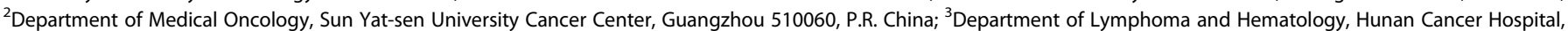

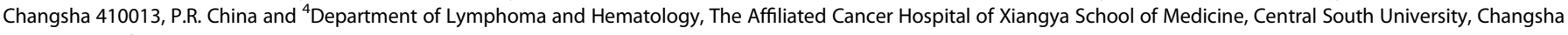
410013, P.R. China

Correspondence: Qingqing Cai (caiqq@sysucc.org.cn)

These authors contributed equally: Jun Cai, Panpan Liu, Huiqiang Huang
}

Received: 15 July 2020 Revised: 1 September 2020 Accepted: 13 September 2020

Published online: 30 December 2020 

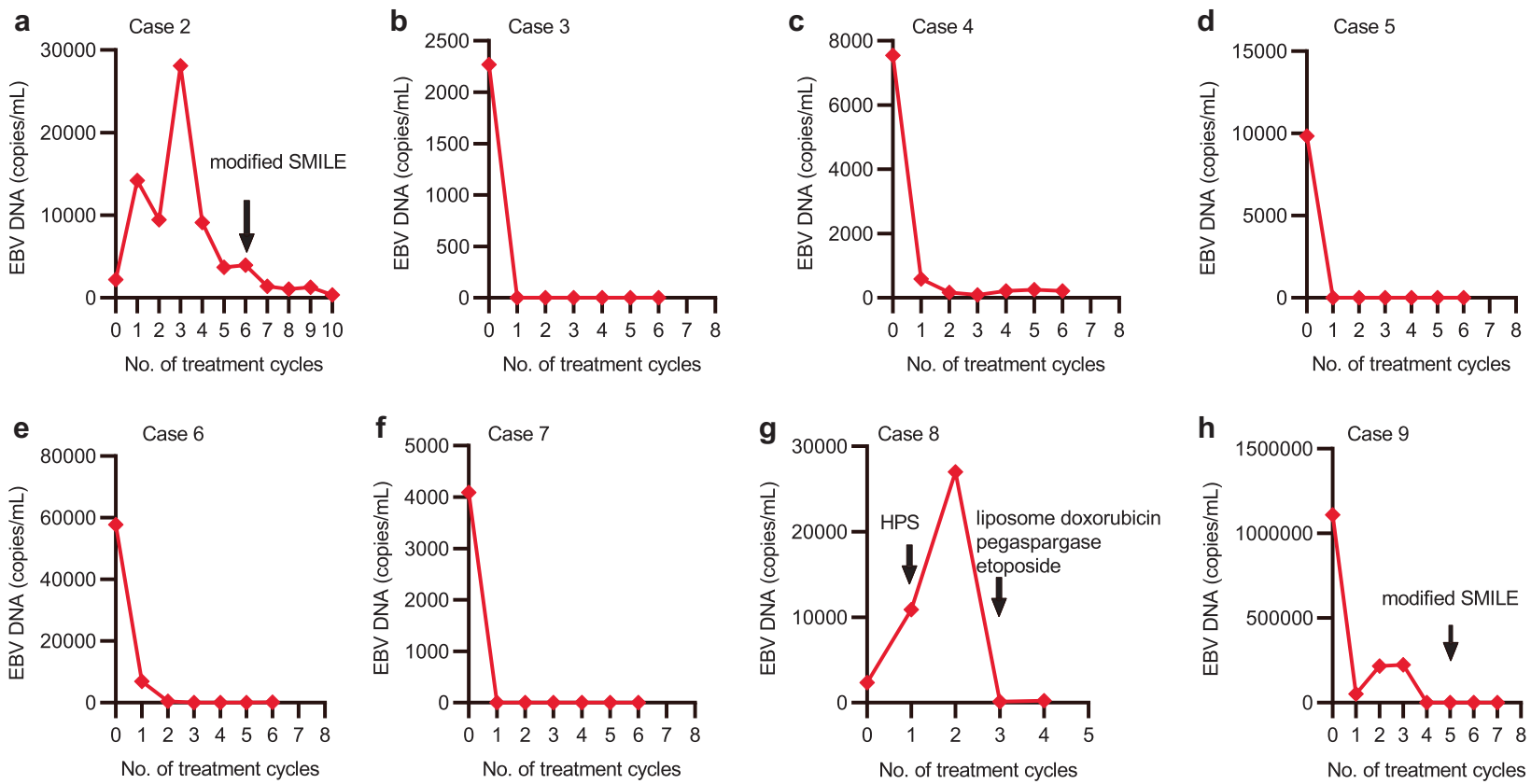

Fig. 1 Changes in circulating EBV DNA during immunochemotherapy. a The EBV DNA in case 2 increased from 2220 to 28,100 copies/mL after 3 cycles of immunochemotherapy, and then became normal after salvage therapy of modified SMILE. b-f The EBV DNA in cases 3, 4, 5, 6, and 7 became normal after 1 or 2 cycles of immunochemotherapy. g The EBV DNA in case 8 increased from 2350 to 27,000 copies/mL after immunochemotherapy, and became normal after salvage therapy of etoposide, pegaspargase, and liposome doxorubicin. $\mathbf{h}$ The EBV DNA in case 9 decreased after the first cycle of immunochemotherapy, and then increased. EBV DNA then fell to 0 copies $/ \mathrm{mL}$ after salvage therapy of modified SMILE

(EBV), ${ }^{17,18}$ making NKTL a target for anti-programmed death 1 (anti-PD-1)/PD-L1 antibodies. ${ }^{19,20}$ Several studies have reported that the single-agent anti-PD-1 antibody could provide an ORR of $57.1-100 \%$ in $\mathrm{r} / \mathrm{r}$ NKTL, with a 1-year OS rate of $82.1 \% .^{21-25}$ Further, those encouraging results on anti-PD-1 antibody in NKTL have started to challenge the current treatment paradigms of NKTL and have provided the rationale for evaluating PD-1 blockade as a firstline therapy of patients with advanced NKTL.

More recently, as first-line therapy, anti-PD-1 antibody combined with chemotherapy has shown benefits in solid tumors. In the KEYNOTE-407 trial, treatment with pembrolizumab plus chemotherapy was found to be superior than chemotherapy alone for squamous non-small-cell lung cancer in terms of PFS and OS. ${ }^{26}$ In the KEYNOTE-048 study, pembrolizumab plus chemotherapy demonstrated superior OS compared with cetuximab plus chemotherapy for recurrent or metastatic head and neck squamous cell carcinoma. ${ }^{27}$

Considering the potential synergistic efficacy of immune checkpoint inhibitor and chemotherapy, we propose a novel treatment strategy by combining anti-PD-1 antibody with PGEMOX for advanced NKTL and herein report its treatment efficacy, safety, and exploratory biomarker results.

\section{RESULTS}

Patients

A total of nine patients diagnosed with advanced NKTL were treated and included in this study. Their median age was 38 (range, 22-65) years. Circulating EBV DNA ranged from 2220 to $1,110,000$ copies/mL (Fig. 1) and Epstein-Barr virus-encoded RNA (EBER) was pathologically confirmed as positive in all cases. The patients' characteristics are summarized in Table 1.

Response to the anti-PD-1 antibody plus P-GEMOX regimen After a median of 5 (range, 1-7) cycles of the prescribed proposed immunochemotherapy regimen, objective response was observed in eight patients. The ORR for the nine treated patients was $88.9 \%$, including 7 CRs (77.8\%) and 1 partial response (PR, 11.1\%) (Table 2 and Supplementary Fig. 1). The 1-year PFS rate was $66.7 \%$ and the 1 -year OS rate was $100 \%$ (Supplementary Fig. 2).

We first explored the potential impact of the immunochemotherapy regimen in a woman presented with nasopharyngeal and systemic lymph node involvement. She showed no response to P-GEMOX and P-GEMOX plus chidamide therapy. Then, anti-PD1 antibody was given as a combination with the P-GEMOX regimen. All the lesions responded well, and a CR was observed at the end of the second course of the immunochemotherapy regimen (Fig. 2a-f). We next evaluated the immunochemotherapy regimen in another eight advanced patients, of whom seven demonstrated promising responses ( $C R$, six patients; $P R$, one patient). Images of two patients (cases 3 and 6) with objective responses are shown in Fig. $2 \mathrm{~g}-\mathrm{I}$.

After a median follow-up of 10.6 (range, 7.5-21.9) months, six patients remained in $\mathrm{CR}$, and three experienced disease progression after achieving their best response. Case 2 achieved a CR after 4 cycles of immunochemotherapy, but before the fourth cycle, there was an increase in EBV DNA (Fig. 1a) and then bone marrow aspiration showed tumor cells reappearance in the bone marrow. The PFS of case 2 was 3.8 months. Case 9 presented with involvement of the nasopharynx, systemic bones, and lymph nodes of the neck and axilla. She developed hemophagocytic syndrome (HPS) during the first cycle of treatment. And then, her EBV DNA decreased (Fig. 1h), HPS was resolved and positron emission tomography-computed tomography (PET/CT) confirmed a PR after 2 cycles of immunochemotherapy. However, after 4 cycles of immunochemotherapy, her EBV DNA was found to be increased and PET/CT provided confirmatory evidence of disease progression. The PFS for case 9 was 2.9 months. Case 8 showed recurrent fever and facial midline destructive lesions, with PET/CT demonstrating involvement of the nasal cavities, sinuses, tonsils, and cervical lymph nodes. After the first cycle of immunochemotherapy, the EBV DNA significantly increased (Fig. 1g), HPS occurred, and the facial 


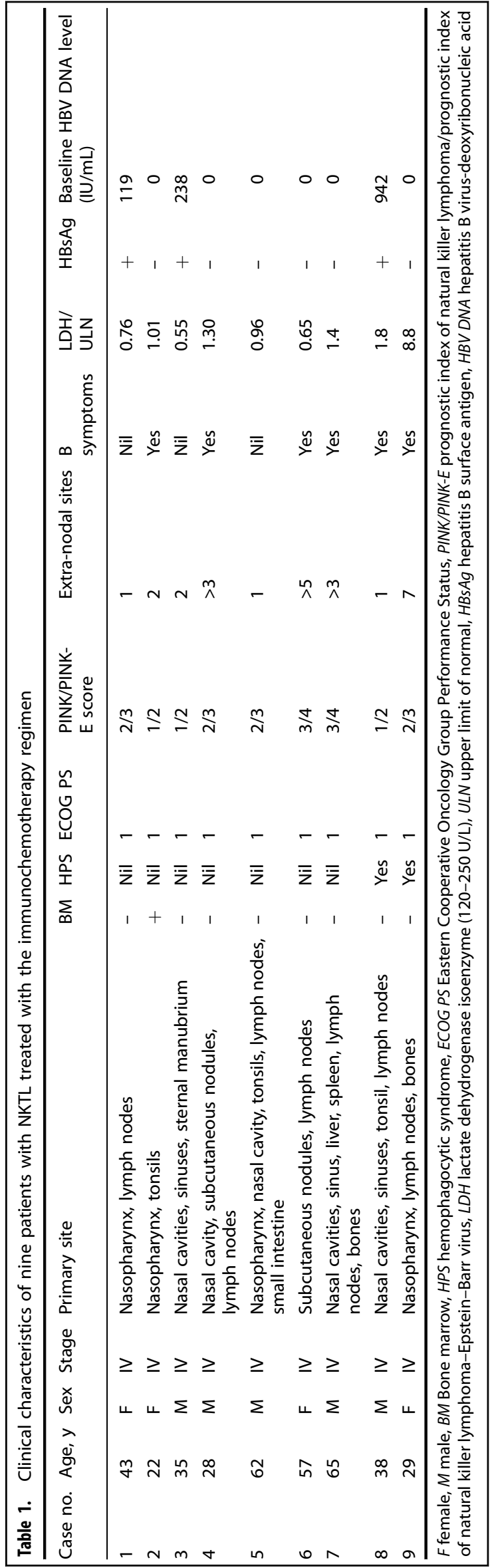

lesions progressed. The patient was then prescribed with the etoposide, pegaspargase, and liposome doxorubicin treatment. After 1 cycle, the HPS resolved, the facial lesions improved, and EBV DNA became undetectable (Fig. 1g). During anti-PD-1 antibody maintenance, two patients experienced pseudoprogression. The related details are shown in Supplementary Materials.

Adverse events

All nine patients experienced treatment-related AEs. The most common grade 3 or 4 AEs were anemia (3 [33.3\%] patients), neutropenia (3 [33.3\%] patients), and thrombocytopenia (3 [33.3\%] patients). Only one patient (case 4) developed immunemediated AE of grade 2 hypothyroidism (Supplementary Table 1). No patients discontinued the treatment because of treatmentrelated AEs and all the AEs were manageable and resolved. Three patients with hepatitis B virus (HBV) infection received oral antiviral treatment (entecavir) and regular monitoring of HBV DNA. No patients had HBV outbreak or reactivation during the treatment. At data cutoff, their HBV DNA load all dropped to $0 \mathrm{IU} / \mathrm{mL}$ with the continuous and effective antiviral therapy.

Expression of PD-L1

Immunohistochemistry (IHC) examination of PD-L1 expression and pathological markers are shown in Fig. 3 and Supplementary Table 2. Six (85.7\%) patients (cases 2, 3, 4, 5, 6, and 8) demonstrated positivity of PD-L1 expression (Table 2). Five of them responded to immunochemotherapy and attained CR. Only one patient (case 8) with a high expression of PD-L1 was insensitive to immunochemotherapy. An absence (case 9) of tumor PD-L1 expression was found correlated with inferior response. Data were not available for PD-L1 IHC analysis in two patients (cases 1 and 7).

Targeted next-generation sequencing-based mutation profiling of tissues

As mentioned above, not all patients with positive PD-L1 expression responded to the immunochemotherapy regimen. To further explore the molecular mechanism of immunochemotherapy in advanced NKTL, 446 lymphoma- and cancer-relevant genes using next-generation sequencing (NGS) were analyzed in seven patients with available tumor tissues. Figure 4 shows the heatmap of their gene mutation profiles. The majority of gene alterations were missense mutations and a dominant $\mathrm{G}: \mathrm{C}$ to $\mathrm{A}: \mathrm{T}$ transition (Supplementary Fig. 3). The top five most frequently mutated genes were STAT3 (cases 4, 6, 9; 42.9\%, 3/7), KMT2D (cases 4, 5, 9; 42.9\%, 3/7), ARID1A (cases 2, 6, 9; 42.9\%, 3/7), BCOR (cases 2, 3, 6; $42.9 \%, 3 / 7$ ), and TET2 (cases 6, 8, 9; 42.9\%, 3/7). Mutations for DDX3X was $28.6 \%$ (cases 2,$8 ; 2 / 7$ ), TP 53 was $28.6 \%$ (cases 3,$4 ; 2$ / 7 ), and CD274/PD-L1 was $14.3 \%$ (case $5,1 / 7$ ). Among the above changes, the STAT3 activating mutation in cases 4 and 6 was associated with PD-L1 overexpression and good response, whereas no PD-L1 expression was detected in case 9 who exhibited poor response. ARID1A mutations (STOP-gained and frame-shift) were observed in cases 2,6 , and 9 . The two patients (cases 2 and 8) who had DDX3X mutations experienced disease progression. The third patient with poor clinical outcome (case 9) exhibited mutations mainly at the epigenetic modifiers, as previously defined. ${ }^{28}$ Gene Ontology-Biological Process (GO-BP) enrichment analysis revealed that the T-cell receptor signaling pathway, immune response-regulating pathway, and immune response-activating pathway were enriched in the group without disease progression (cases 3, 4, 5, and 6) (Supplementary Fig. 4).

\section{DISCUSSION}

This is the first time that the role of immune checkpoint inhibition plus pegaspargase-based chemotherapy in advanced NKTL has been evaluated. Our results suggested that the addition of an antiPD-1 antibody to the P-GEMOX regimen could be a highly 
Table 2. Therapies and outcomes of nine patients with NKTL treated with the immunochemotherapy regimen

\begin{tabular}{|c|c|c|c|c|c|c|}
\hline Case no. & TPS & CPS & Treatment regimens (cycles) & $\begin{array}{l}\text { Anti-PD-1 antibody } \\
\text { dose, mg }\end{array}$ & $\begin{array}{l}\text { Best overall } \\
\text { response }\end{array}$ & PFS, months \\
\hline 1 & NA & NA & $\begin{array}{l}\text { P-GEMOX (4), P-GEMOX+Chidamide (2), P-GEMOX+Pembrolizumab } \\
\text { (2), Sintilimab (8) }\end{array}$ & 200 & $\mathrm{CR}$ & 15.5 \\
\hline 2 & 8 & 10 & P-GEMOX (1); P-GEMOX+Toripalimab (4) & 240 & CR & 3.8 \\
\hline 3 & 30 & 45 & $\begin{array}{l}\text { P-GEMOX (1); P-GEMOX+Sintilimab (5); sequential RT with } \\
\text { concurrent Sintilimab (2); Sintilimab (5) }\end{array}$ & 200 & $\mathrm{CR}$ & 14.0 \\
\hline 4 & 40 & 45 & $\begin{array}{l}\text { P-GEMOX+Sintilimab (3); } \\
\text { P-GEMOX+Camrelizumab (3) }\end{array}$ & $\begin{array}{l}200 \\
200\end{array}$ & CR & 11.8 \\
\hline 5 & 98 & 100 & $\begin{array}{l}\text { P-GEMOX (1); P-GEMOX+Sintilimab (1); } \\
\text { P-GEMOX (2) with sequential RT; P-GEMOX (3) }\end{array}$ & 200 & CR & 10.6 \\
\hline 6 & 10 & 13 & P-GEMOX+Sintilimab (6); Sintilimab (1) & 200 & $\mathrm{CR}$ & 9.4 \\
\hline 7 & NA & NA & P-GEMOX+Sintilimab (6) & 200 & CR & 7.5 \\
\hline 8 & 50 & 55 & P-GEMOX+Sintilimab (1) & 200 & PD & 0.8 \\
\hline 9 & 0 & 0 & P-GEMOX (2); P-GEMOX + Sintilimab (2) & 200 & PR & 2.9 \\
\hline
\end{tabular}

TPS tumor proportion score, the number of PD-L1 positive tumor cells (showing partial or complete membrane PD-L1 staining at any intensity) divided by the total number of tumor cells $\times 100$; CPS combined positive score, the number of PD-L1 positive cells (tumor cells, lymphocytes, macrophages) divided by the total number of tumor cells $\times 100, N A$ not available, $P$-GEMOX pegaspargase, gemcitabine and oxaliplatin, $R T$ radiotherapy, $C R$ complete remission, $P R$ partial remission, $P D$ progression of disease, PFS progress-free survival

effective combination for patients with advanced NKTL and possessed a favorable safety profile. The dynamic change in circulating EBV DNA was associated with therapeutic efficacy, which proved to be a significant predictor of response.

In addition to being a safe and effective regimen for NKTL treatment, P-GEMOX may also have an immune-enhancing effect. Gemcitabine, a nucleoside analog included in the P-GEMOX regimen, has been found to reduce the amount of circulating myeloid-derived suppressor cells (MDSCs), favoring the reprogramming of tumor-associated macrophages toward an immunostimulatory phenotype. ${ }^{29-31}$ Besides such direct immunostimulatory effects, gemcitabine could also stimulate the expression of major histocompatibility complex (MHC) class I molecules in cancer cells, thereby increasing their antigenicity. ${ }^{32}$ Oxaliplatin (a platinum derivative also included in the P-GEMOX regimen) has been shown to be able to promote immunogenic cell death by increasing the $\mathrm{CD}^{+}$cytotoxic $\mathrm{T}$ lymphocytes $(\mathrm{CTLs}) / \mathrm{CD} 4^{+} \mathrm{CD} 25^{+} \mathrm{FOXP}^{+}$regulatory $\mathrm{T}\left(\mathrm{T}_{\mathrm{REG}}\right)$ cell ratio and depleting MDSCs, as well as promoting the activity of neutrophils and macrophages. Thereby, oxaliplatin treatment can generate a robust effector immune response. ${ }^{33,34}$ Combining immunogenic P-GEMOX chemotherapy with PD-1 blockade theoretically drives deeper and sustained tumor responses. In this present study, 4 types of anti-PD-1 antibodies were used. Although bonded to different target epitopes at the PD-1 molecule, ${ }^{35-38}$ they had the same mechanism of action (inhibiting the PD-1/PD-L1 immune checkpoint, leading to restoration of T-cell functions against cancer cells), and thus would not significantly affect the interpretation of the study results. This notion is supported by our observation that eight out of the nine investigated patients treated with different anti-PD-1 antibodies plus P-GEMOX showed good clinical responses, including 7 CRs and 1 PR.

The major advantage of this regimen is that it does not require hematopoietic stem cell transplantation (HSCT) as a postremission consolidation therapy. The role of HSCT in NKTL has been controversial. Evidence supporting the use of HSCT (autologous-HSCT or allogenic-HSCT) is mainly based on the results of uncontrolled retrospective studies, and there are no randomized studies that directly compared post-remission consolidation HSCT versus observation for NKTL. Survival benefits from HSCT were suggested by a retrospective analysis that compared 47 patients who underwent autologous-HSCT with 107 historical controls. ${ }^{39}$ For patients who attained $\mathrm{CR}$, the 5-year disease-specific survival of HSCT group was higher than controls ( $87.3 \%$ versus $67.8 \%$ ). However, conclusions should be interpreted with caution because the study did not match the groups for the chemotherapeutic regimen or timing of radiotherapy. Another study that included 21 patients with advanced disease reported no apparent difference in long-term outcomes between autologous-HSCT group versus historical controls without postremission treatment. ${ }^{40,41}$ Given the high toxicity and uncertainty of efficacy in NKTL consolidation, HSCT was not performed in our study. Besides, prior data showed that the anti-PD-1 antibody exhibited durable antitumor activity in patients receiving this therapy, which persisted after drug discontinuation. Thus, the patients in our study received prolonged anti-PD-1 antibody maintenance aiming to induce long-term remission and survival.

Our study had a CR rate of $77.8 \%$. In previously reported studies on asparaginase or pegaspargase-containing regimens, such as the SMILE and AspaMetDex studies, ${ }^{12,16}$ the observed CR rates in advanced NKTL were $40.0 \%$ and $38.5 \%$, respectively. The CR rate of our immunochemotherapy seems to be better than that of SMILE and AspaMetDex, however, future randomized controlled trials are still needed to verify this conclusion. In a recent randomized controlled multicenter study reported by Huang et al., ${ }^{16} 31$ patients with stage III/IV or r/r NKTL achieved an ORR of $87.1 \%$ and a CR rate of $56.3 \%$ after P-GEMOX plus thalidomide therapy. Attaining a CR has been found to be a significant factor impacting on the outcome of NKTL. In our present study, $77.8 \%$ of patients achieved $C R$, suggesting a better outcome could be observed during future long-term follow-up.

The immunochemotherapy regimen demonstrated an acceptable safety profile in patients with advanced NKTL. The most common AEs in our study were emesis (44.4\%), elevated transaminase (44.4\%), nausea (33.3\%), prolonged activated partial thromboplastin time (APTT, 33.3\%), grade 3 or 4 AEs including anemia (33.3\%), neutropenia (33.3\%), and thrombocytopenia (33.3\%). The hematologic toxicities seen in our study were slightly higher than that reported with the P-GEMOX regimen, which rendered $24.5 \%$ of grade 3 or 4 neutropenia, $10.6 \%$ of grade 3 or 4 anemia, and $15.8 \%$ of grade 3 or 4 thrombocytopenia. ${ }^{16}$ Great importance should be attached to hematologic toxicities related to this regimen. Neither pneumonia nor allergy was observed. No increase in hepatocyte transaminase due to virus replication or reactivation occurred in the three patients with $\mathrm{HBV}$ infection. In 


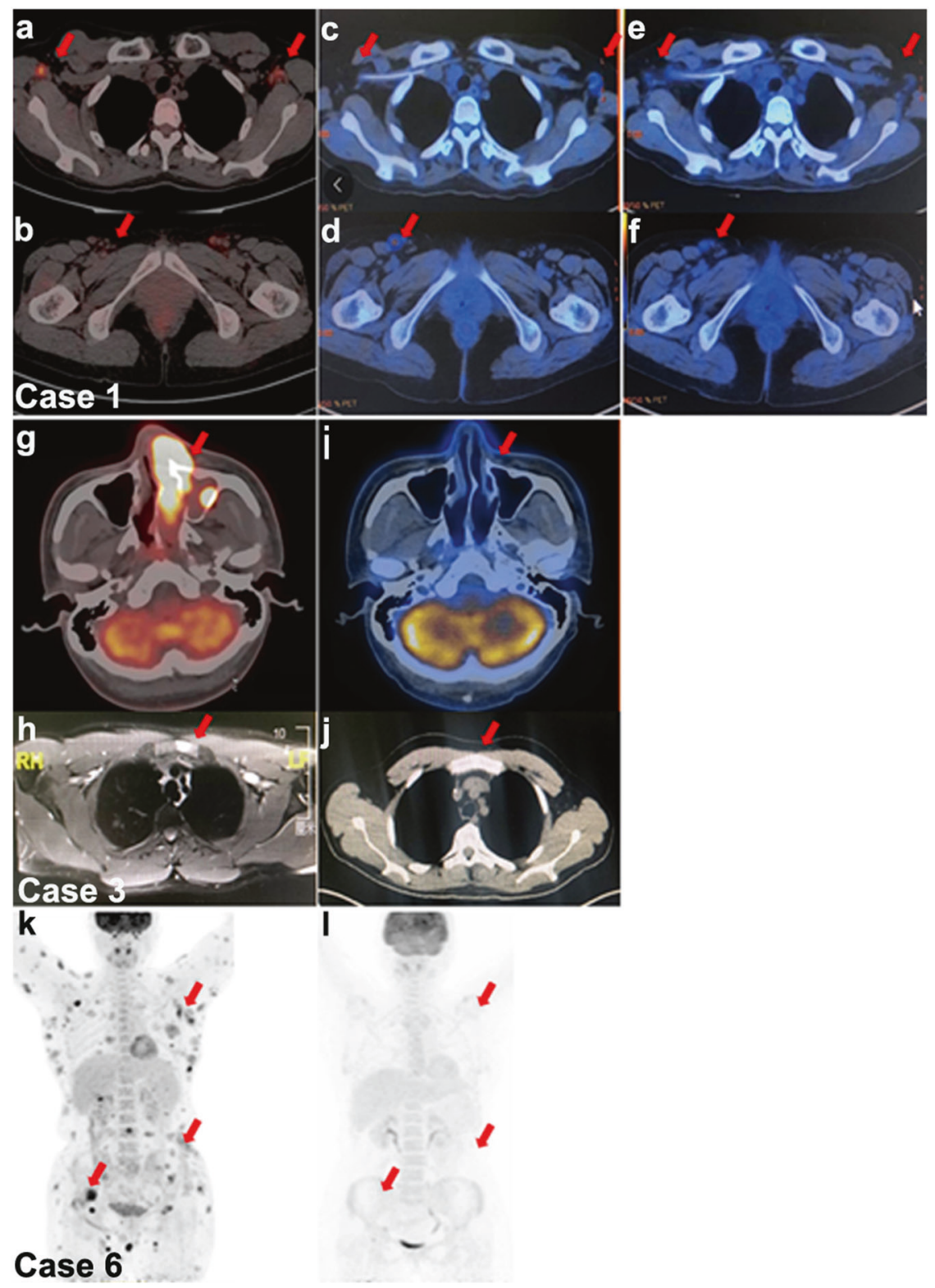

Fig. 2 Imaging results of three representative patients. a-f PET/CT results of case 1. Baseline PET/CT showed multiple lymph nodes involvement $(\mathbf{a}, \mathbf{b})$. After the sixth cycle of conventional chemotherapy, there were persistent FDG-avid lesions by PET/CT scan (c, d). After 2 cycles of immunochemotherapy, no FDG-avid lesions were observed and CR was confirmed (e, f). g-j PET/CT and MRI results of case 3. Baseline PET/CT and MRI examination showed neoplastic metabolic lesions invading the nasal cavities and sinuses, with MRI-proved lymphomatous infiltration in the sternal manubrium $(\mathbf{g}, \mathbf{h})$. After 4 cycles of immunochemotherapy, no FDG-avid lesions were found in a follow-up PET/CT scan, and the manubrium sternum lesions completely disappeared; demonstrating CR (i, j). $\mathbf{k}$, I PET/CT results of case 6 . Baseline PET/CT scan of case 6 , presenting no nasal disease but systemic tumor invasion of the skin and lymph nodes (k). After 6 cycles of immunochemotherapy, all original skin lesions resolved, and no FDG-avid lesions were seen in the PET/CT scan, which indicated a response of CR (I)

the present study, no discontinuation or death caused by treatment-related AEs was observed and all the AEs were manageable and reversible.

Apart from the exploration of the most efficient combination of therapy, identification of biomarkers to select patients who could benefit from PD-1 inhibition is also required. PD-L1 expression results were evaluable in seven patients, of whom six were found positive. PD-L1 positive patients had a higher response rate $(C R$, $n=5$ ). Case 9 , who was refractory to the immunochemotherapy regimen, showed no detectable PD-L1 expression. Dynamic monitoring of circulating EBV DNA value suggested that EBV DNA copy number was associated with the tumor activity and predictive of the treatment efficacy. During the course of the immunochemotherapy regimen, a significant reduction in plasma EBV DNA was observed in cases $3,4,5,6$, and 7. A rapid increase in plasma EBV DNA levels was associated with disease progression, while a decrease was associated with the control of the disease; which was observed in cases 2,8 , and 9.

This study is the first to have explored genes alteration in advanced NKTL and their potential roles as biomarkers in response to PD-1 inhibitor combined chemotherapy treatment. Our NGS results suggested that the STAT3/JAK3/PD-L1 alterations were associated with immunochemotherapy. This is consistent with the observation that STAT3 mutation could increase the phosphorylation 


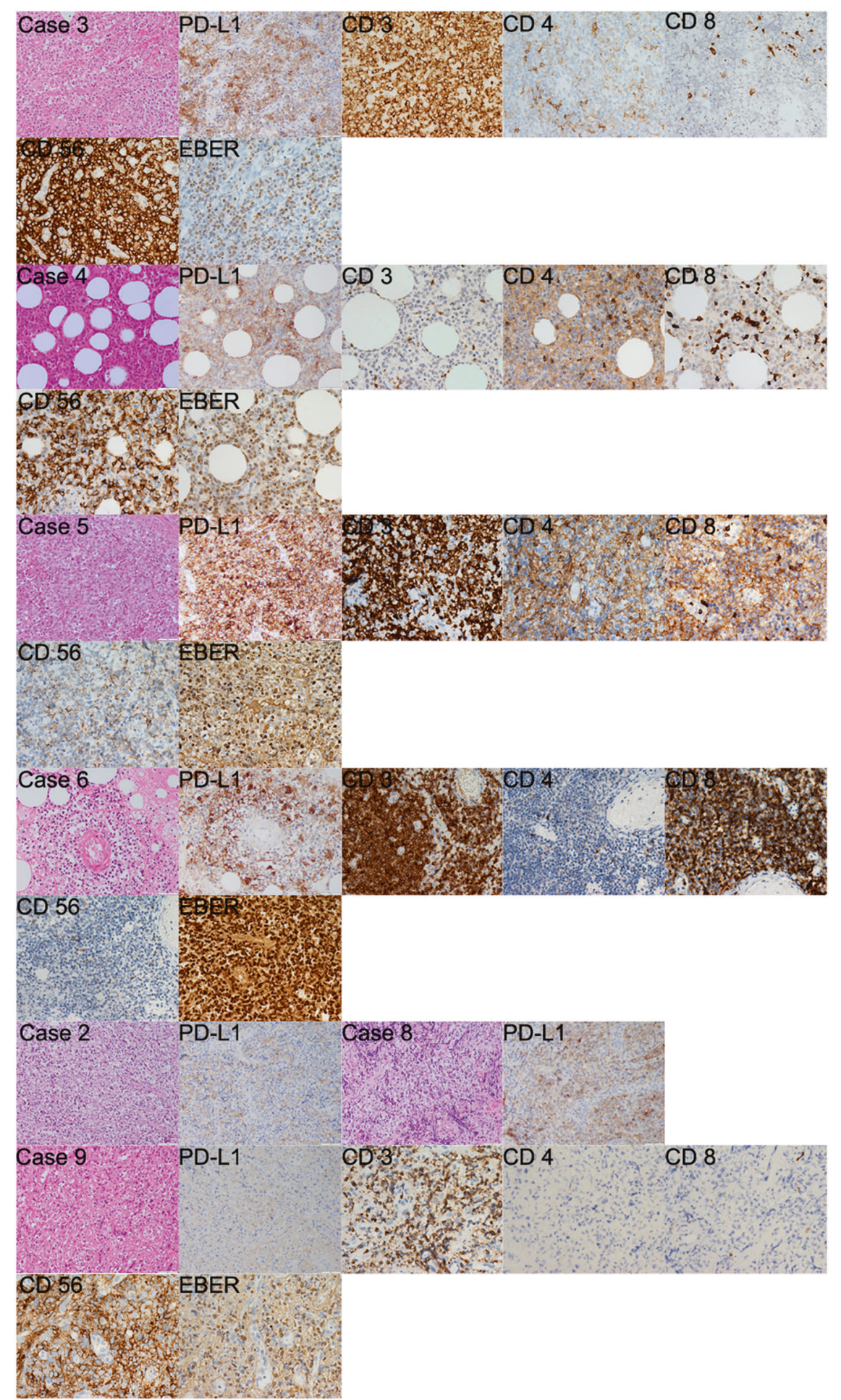

Fig. 3 Immunohistochemistry (IHC) and hematoxylin/eosin (HE) staining of tumor specimens from seven NKTL patients. The expression of PDL1, CD3, CD4, CD8, CD56, and Epstein-Barr virus-encoded RNA (EBER) in the clinical samples was analyzed by IHC staining (original magnification $\times 400$ ). Due to limited tissue slides available for immunostaining, some of the specimens were only stained for the selected markers as indicated. PD-L1 was negative for case 9. EBER was positive in all seven cases. The expression of PD-L1 was only stained for cases 2 and 8

of STAT3, and could thus enhance the transcription activity of STAT3, leading to elevated PD-L1 expression by binding to the promoter of PD-L1 gene. ${ }^{42}$ Recently, a study ${ }^{28}$ stratified NKTL into 3 molecular subtypes using multi-omics analysis. In this present study, case 9 had negative PD-L1 expression (IHC score, 0). Mutations in
ARID1A, KMT2D, TET2, and BCORL1 for case 9 suggested deregulation of the epigenetic control of transcription. This patient seemed to belong to the HEA subtype ${ }^{28}$ and was more sensitive to the histone deacetylase inhibitors. ARID1A is a tumor suppressor gene and several studies showed that ARID1A mutations were associated with 


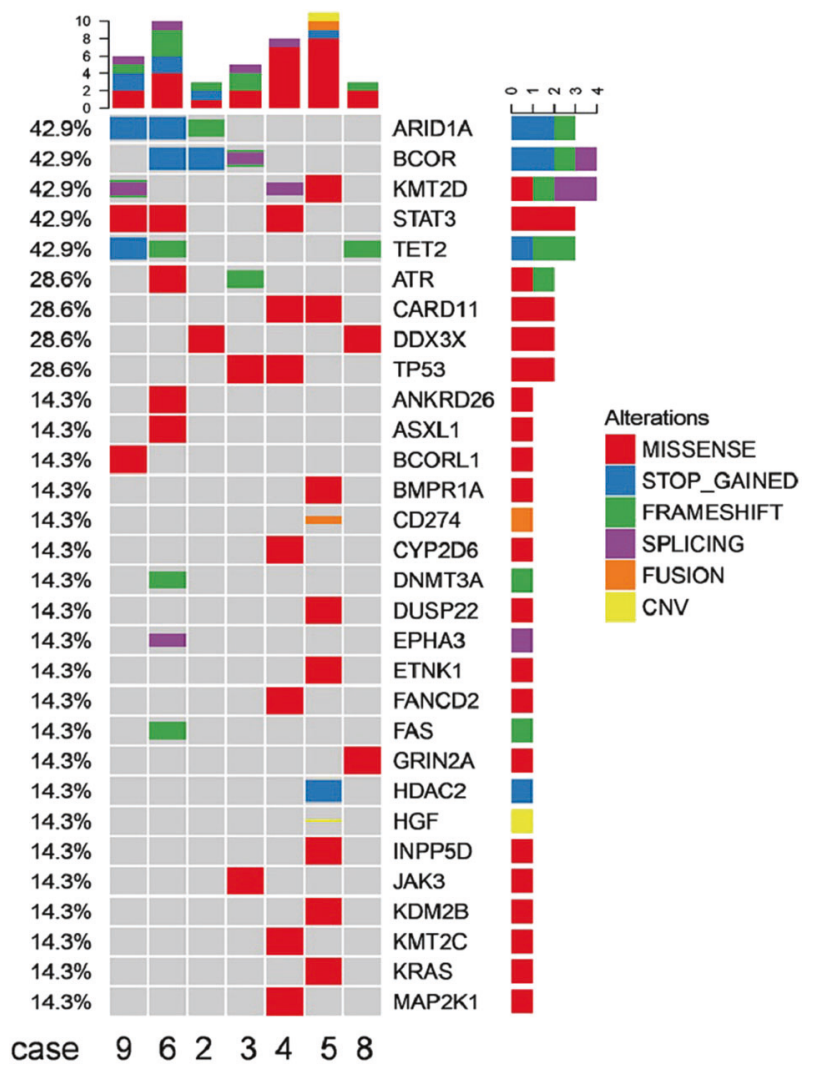

Fig. 4 Heatmap for altered genes in the seven NKTL patients

increased immune activity and could predict benefits from checkpoint blockade in solid tumors. ${ }^{43-45}$ In our study, case 9 was negative for PD-L1 expression but also responded to the immunochemotherapy, suggesting that such positive response might be attributed to ARID1A alteration. Case 8 had higher expression of PD-L1 but was resistant to PD-1 inhibition. This negative result could be due to $D D X 3 X$ mutation since it was previously shown that $D D X 3 X$ mutation was associated with poor prognosis. ${ }^{28,46}$ Further, a recent study showed that $D D X 3 X$ mutation could affect cell-fate decisions in cells under stress conditions by regulating NLRP3 inflammasome. ${ }^{47}$ In our study, the DDX3X mutation might change the response of NKTL to the stress induced by anti-PD-1 antibody and P-GEMOX, leading to the blocking of lymphoma cell death and drug resistance. Overall, our findings suggested that mutations in STAT3, ARID1A, and DDX3X might be important molecular events that could be combined with PD-L1 expression to predict clinical response to immunochemotherapy.

There were several limitations worth mentioning. First, the investigated cohort comprised of a relatively small sample size. Second, the biomarker analysis of PD-L1 expression and tumor mutations could have been insufficiently powered due to the limited biopsy samples available. Third, the antitumor activity analyses were preliminary. Thus, a larger sample size prospective study with longer follow-up time is still required for biomarker, PFS, and OS assessment to validate the study's findings.

Based on the results of this exploratory study, we initiated a multicenter, single-arm, phase II trial (NCT04127227) consisting of sintilimab combined with P-GEMOX for untreated advanced NKTL patients. Future results of this prospective study could shed more light on the efficacy and safety profile of immunochemotherapy in this patients' category. Molecular biology and genetic analysis could further help to differentiate patients based on their predicted response to immunochemotherapy.

\section{MATERIALS AND METHODS}

Patients and treatment

From July 6, 2018, to September 11, 2019, a total of nine patients with advanced NKTL received anti-PD-1 antibody with P-GEMOX at the Sun Yat-sen University Cancer Center (Guangzhou, China) and Hunan Cancer Hospital (Changsha, China). Patients with advanced NKTL received six cycles of anti-PD-1 antibody (day 1), pegaspargase $2000 \mathrm{U} / \mathrm{m}^{2}$ (day 1 ), gemcitabine $1 \mathrm{~g} / \mathrm{m}^{2}$ (days 1 and 8), and oxaliplatin $130 \mathrm{mg} / \mathrm{m}^{2}$ (day 1) every 3 weeks. Those who achieved an objective response received anti-PD-1 antibody maintenance once every 3 weeks (Supplementary Fig. 5). Eligible patients were aged 18 years and older, and had adequate organ and bone marrow function. Patients with central nervous system involvement and secondary tumors were excluded. Patients with HBV infection were required to be receiving effective and continuous antiviral therapy (entecavir), and have a viral load of $<1000 \mathrm{IU} / \mathrm{mL}$ at baseline.

Response assessment

Scheduled ${ }^{18} \mathrm{~F}$-fluorodeoxyglucose $\left({ }^{18} \mathrm{FDG}\right) \mathrm{PET} / \mathrm{CT}$ or magnetic resonance imaging (MRI) was performed. Plasma samples were collected every 3 weeks to dynamically monitor circulating EBV and HBV load by quantitative polymerase chain reaction (PCR). Response assessment was made every 6 weeks according to standard criteria (5-point Deauville score). ${ }^{48}$ During anti-PD-1 antibody maintenance therapy, response assessment was made every 3 months using the Lymphoma Response to Immunomodulatory Therapy Criteria (LYRIC). ${ }^{49}$ The National Cancer Institute Common Terminology Criteria for Adverse Events, version 5.0 was used to grade AEs.

Immunohistochemistry analysis

Paraffin-embedded pretreatment tissue samples were obtained from seven patients. Expression of tumoral PD-L1 was determined by $\mathrm{IHC}$ using mouse monoclonal antibody clone $22 \mathrm{c} 3$. Combined positive score (CPS) was defined as the number of PD-L1 positive cells (tumor cells, lymphocytes, macrophages) divided by the total number of tumor cells $\times 100$; Tumor proportion score (TPS) was defined as the number of PD-L1 positive tumor cells (showing partial or complete membrane PD-L1 staining at any intensity) divided by the total number of tumor cells $\times 100$. An expression percentage of $5 \%$ was applied as the threshold cutoff for PD-L1 positivity. ${ }^{50}$

Next-generation sequencing analysis

Genomic DNA from formalin-fixed paraffin-embedded tissue sections was extracted with QIAamp DNA FFPE Tissue kit (Qiagen). Sequencing libraries were prepared using the KAPA Hyper Prep Kit (KAPA Biosystems) according to manufacturer's instructions for different sample types. Customized $\mathrm{xGen}$ lockdown probes (Integrated DNA Technologies) targeting 446 leukemia- and lymphoma-related genes were used for hybridization enrichment. Capture reaction was performed with Dynabeads M-270 (Life Technologies) and xGen Lockdown hybridization and wash kit (Integrated DNA Technologies) according to manufacturers' protocols. Genomic DNA was extracted for NGS analysis. All samples subjected to NGS analysis were required to have $>10 \%$ of tumor cells as identified by immunohistochemistry.

\section{Sequencing data analyses}

Mutation Calling Trimmomatic was used for FASTQ file quality control. Leading/trailing low quality (quality reading below 20) or $\mathrm{N}$ bases were removed. Paired-end reads were then aligned to the reference human genome (build hg19) using the Burrows-Wheeler Aligner (BWA). PCR deduplication was performed using Picard and local realignment around indels and base quality score recalibration were performed using GATK3. Unfortunately, matched germline DNA of patients as a normal control for mutation analysis was not 
available for this retrospective study. Somatic mutations were first called for each sample (the filtering criteria were a variant frequency of $\geq 0.5 \%$ and 5 or more supporting reads from both directions). Common single-nucleotide polymorphisms were excluded if they were present in $>1 \%$ of the population frequency in the 1000 Genomes Project or the Exome Aggregation Consortium 65,000exome database. The resulting mutation list was further filtered by an in-house list of recurrent artifacts and common single-nucleotide polymorphisms based on $~ 500$ whole blood samples (normal pool) from Chinese patients with cancer that were sequenced with the same gene panel at an average depth of 400x. All SNVs/indels were annotated with ANNOVAR, and each SNV/indel was manually checked on the Integrative Genomics Viewer (IGV). Copy number variations (CNVs) were detected using in-house-developed software.

\section{Ethical consideration}

All patients were informed of possible AEs and provided informed consent for the treatment. The study was approved by the Institutional Review Board of Sun Yat-sen University Cancer Center (No. B2020-163-01).

\section{DATA AVAILABILITY}

The authenticity of this article has been validated by uploading the key raw data onto the Research Data Deposit public platform (www.researchdata.org.cn), with the approval RDD number as RDDA2020001527.

\section{ACKNOWLEDGEMENTS}

This work was supported by the National Natural Science Foundation of China (81672686), Special Support Program of Sun Yat-sen University Cancer Center (PT19020401), Science and Technology Planning Project of Guangzhou, China (202002030205), and Clinical Oncology Foundation of Chinese Society of Clinical Oncology (Y-XD2019-124).

\section{AUTHOR CONTRIBUTIONS}

J.C., P.L., and H.H. contributed to study design, statistical analysis, data collecting, figure, and tables preparation. Y.L., S.M., H.Z., X.T., Y.Z., Y.G., Y.X., X.Z., H.Y., and L.L. performed sample preparation for immunohistochemistry and next-generation sequencing analysis and clinical research. J.C., P.L., and Q.C. performed manuscript writing and review. All authors agree with the contents of this paper.

\section{ADDITIONAL INFORMATION}

The online version of this article (https://doi.org/10.1038/s41392-020-00331-3) contains supplementary material, which is available to authorized users.

Competing interests: The authors declare no competing interests.

\section{REFERENCES}

1. Cai, Q., Cai, J., Fang, Y. \& Young, K. H. Epstein-Barr virus-positive natural killer/Tphoma. Front. Oncol. 9, 386 (2019).

2. Haverkos, B. M. et al. Extranodal NK/T cell lymphoma, nasal type (ENKTL-NT): an update on epidemiology, clinical presentation, and natural history in North American and European cases. Curr. Hematol. Malig. Rep. 11, 514-527 (2016).

3. Kim, T. M. et al. Clinical heterogeneity of extranodal NK/T-cell lymphoma, nasal type: a national survey of the Korean Cancer Study Group. Ann. Oncol. 19, 1477-1484 (2008).

4. Au, W.-y et al. Clinical differences between nasal and extranasal natural killer/Tcell lymphoma: a study of 136 cases from the International Peripheral T-Cell Lymphoma Project. Blood 113, 3931-3937 (2009).

5. Yang, Y. et al. Risk-adapted therapy for early-stage extranodal nasal-type NK/Tcell lymphoma: analysis from a multicenter study. Blood 126, 1424-1432 (2015).

6. Yang, Y. et al. Association of improved locoregional control with prolonged survival in early-stage extranodal nasal-type natural killer/T-cell lymphoma. JAMA Oncol. 3, 83-91 (2017).

7. Kwong, Y. L. et al. Sequential chemotherapy/radiotherapy was comparable with concurrent chemoradiotherapy for stage I/IINK/T-cell lymphoma. Ann. Oncol. 29, 256-263 (2018)
8. Yang, Y. et al. Prognostic nomogram for overall survival in previously untreated patients with extranodal NK/T-cell lymphoma, nasal-type: a multicenter study. Leukemia 29, 1571-1577 (2015).

9. Fox, C. P. et al. Survival outcomes of patients with extranodal natural-killer T-cell lymphoma: a prospective cohort study from the international T-cell Project. Lancet Haematol. 7, e284-e294 (2020).

10. Yamaguchi, M. et al. Treatments and outcomes of patients with extranodal natural killer/T-cell lymphoma diagnosed between 2000 and 2013: a cooperative study in Japan. J. Clin. Oncol. 35, 32-39 (2017).

11. Wang, L. et al. IL-2R alpha up-regulation is mediated by latent membrane protein 1 and promotes lymphomagenesis and chemotherapy resistance in natural killer/ T-cell lymphoma. Cancer Commun. 38, https://doi.org/10.1186/s40880-018-03348 (2018).

12. Yamaguchi, M. et al. Phase II study of SMILE chemotherapy for newly diagnosed stage IV, relapsed, or refractory extranodal natural killer (NK)/T-cell lymphoma, nasal type: the NK-cell tumor study group study. J. Clin. Oncol. 29, 4410-4416 (2011).

13. Kwong, Y.-L. et al. SMILE for natural killer/T-cell lymphoma: analysis of safety and efficacy from the Asia Lymphoma Study Group. Blood 120, 2973-2980 (2012).

14. Jaccard, A. et al. Efficacy of L-asparaginase with methotrexate and dexamethasone (AspaMetDex regimen) in patients with refractory or relapsing extranodal NK/T-cell lymphoma, a phase 2 study. Blood 117, 1834-1839 (2011).

15. Gao, Y. et al. P-Gemox regimen (pegaspargase, gemcitabine, oxaliplatin) for extranodal natural killer cell lymphoma: 10 years' real-world clinical experience from China. Blood 624, abstr. 1659 (2018).

16. Huang, H. Q. et al. Clinical outcome of an multicentre, randomized, phase II clinical trial for patients with extranodal NK/T cell lymphoma treated by P-Gemox or Aspametdex. Blood 624, abstr. 1569 (2019).

17. Sun, L., Zhao, Y., Shi, H., Ma, C. \& Wei, L. LMP-1 induces survivin expression to inhibit cell apoptosis through the NF-kappa B and PI3K/Akt signaling pathways in nasal NK/T-cell lymphoma. Oncol. Rep. 33, 2253-2260 (2015).

18. Bi, X.-w et al. PD-L1 is upregulated by EBV-driven LMP1 through NF-kappa B pathway and correlates with poor prognosis in natural killer/T-cell lymphoma. J. Hematol. Oncol. 9, 109 (2016).

19. Jo, J.-C. et al. Expression of programmed cell death 1 and programmed cell death ligand 1 in extranodal NK/T-cell lymphoma, nasal type. Ann. Hematol. 96, 25-31 (2017).

20. Nagato, T. et al. Programmed death-ligand 1 and its soluble form are highly expressed in nasal natural killer/T-cell lymphoma: a potential rationale for immunotherapy. Cancer Immunol. Immunother. 66, 877-890 (2017).

21. Kwong, Y.-L. et al. PD1 blockade with pembrolizumab is highly effective in relapsed or refractory NK/T-cell lymphoma failing L-asparaginase. Blood 129, 2437-2442 (2017)

22. $\mathrm{Li}$, X. et al. Activity of pembrolizumab in relapsed/refractory NK/T-cell lymphoma. J. Hematol. Oncol. 11, 15 (2018).

23. Li, J. Y. et al. Sintilimab for relapsed/refractory $(r / r)$ extranodal NK/T cell lymphoma (ENKTL): Extended follow-up on the multicenter, single-arm phase II trail (ORIENT-4). J. Clin. Oncol. 383, abstr. 8050 (2020).

24. Liu, P. et al. Nivolumab treatment of relapsed/refractory Epstein-Barr virus-associated hemophagocytic lymphohistiocytosis in adults. Blood 135, 826-833 (2020).

25. Lai, J., Xu, P., Jiang, X., Zhou, S. \& Liu, A. Successful treatment with antiprogrammed-death-1 antibody in a relapsed natural killer/T-cell lymphoma patient with multi-line resistance: a case report. BMC Cancer 17, 507 (2017).

26. Paz-Ares, L. et al. Pembrolizumab plus chemotherapy for squamous non-smallcell lung cancer. N. Engl. J. Med. 379, 2040-2051 (2018).

27. Burtness, B. et al. Pembrolizumab alone or with chemotherapy versus cetuximab with chemotherapy for recurrent or metastatic squamous cell carcinoma of the head and neck (KEYNOTE-048): a randomised, open-label, phase 3 study. Lancet 394, 1915-1928 (2019).

28. Xiong, J. et al. Genomic and transcriptomic characterization of natural killer T cell lymphoma. Cancer Cell 37, 403-419 e406 (2020).

29. Di Caro, G. et al. Dual prognostic significance of tumour-associated macrophages in human pancreatic adenocarcinoma treated or untreated with chemotherapy. Gut 65, 1710-1720 (2016).

30. Nowak, A. K. et al. Induction of tumor cell apoptosis in vivo increases tumor antigen cross-presentation, cross-priming rather than cross-tolerizing host tumorspecific CD8 T cells. J. Immunol. 170, 4905-4913 (2003).

31. Suzuki, E., Kapoor, V., Jassar, A. S., Kaiser, L. R. \& Albelda, S. M. Gemcitabine selectively eliminates splenic Gr-1+/CD11b+ myeloid suppressor cells in tumorbearing animals and enhances antitumor immune activity. Clin. Cancer Res. 11, 6713-6721 (2005) 
32. Liu, W. M., Fowler, D. W., Smith, P. \& Dalgleish, A. G. Pre-treatment with chemotherapy can enhance the antigenicity and immunogenicity of tumours by promoting adaptive immune responses. Br. J. Cancer 102, 115-123 (2010).

33. Gonzalez-Aparicio, M. et al. Oxaliplatin in combination with liver-specific expression of interleukin 12 reduces the immunosuppressive microenvironment of tumours and eradicates metastatic colorectal cancer in mice. Gut $\mathbf{6 0}$ 341-349 (2011).

34. Tesniere, A. et al. Immunogenic death of colon cancer cells treated with oxaliplatin. Oncogene 29, 482-491 (2010).

35. Lee, J. Y. et al. Structural basis of checkpoint blockade by monoclonal antibodies in cancer immunotherapy. Nat. Commun. 7, 13354 (2016).

36. Zhang, S. et al. Preclinical characterization of Sintilimab, a fully human anti-PD-1 therapeutic monoclonal antibody for cancer. Antib. Ther. 1, 45-53 (2018).

37. Liu, H. et al. Glycosylation-independent binding of monoclonal antibody toripalimab to FG loop of PD-1 for tumor immune checkpoint therapy. MAbs $\mathbf{1 1}$ 681-690 (2019).

38. Markham, A. \& Keam, S. J. Camrelizumab: first global approval. Drugs 79, 1355-1361 (2019).

39. Lee, J. et al. Autologous hematopoietic stem cell transplantation in extranoda natural killer/T cell lymphoma: a multinational, multicenter, matched controlled study. Biol. Blood Marrow Transplant. 14, 1356-1364 (2008).

40. Au, W. Y. et al. Autologous stem cell transplantation for nasal NK/T-cell lymphoma: a progress report on its value. Ann. Oncol. 14, 1673-1676 (2003).

41. Suzuki, R. et al. Hematopoietic stem cell transplantation for natural killer-cell lineage neoplasms. Bone Marrow Transplant. 37, 425-431 (2006).

42. Song, T. L. et al. Oncogenic activation of the STAT3 pathway drives PD-L1 expression in natural killer/T-cell lymphoma. Blood 132, 1146-1158 (2018).

43. Wang, $K$. et al. Exome sequencing identifies frequent mutation of ARID1A in molecular subtypes of gastric cancer. Nat. Genet. 43, 1219-1223 (2011).

44. Shen, J. et al. ARID1A deficiency promotes mutability and potentiates therapeutic antitumor immunity unleashed by immune checkpoint blockade. Nat. Med. 24, 556-562 (2018).
45. Kucuk, C. et al. Activating mutations of STAT5B and STAT3 in lymphomas derived from gamma delta-T or NK cells. Nat Commun 6, https://doi.org/10.1038/ ncomms7025 (2015).

46. Jiang, L. et al. Exome sequencing identifies somatic mutations of DDX3X in natural killer/T-cell lymphoma. Nat. Genet. 47, 1061-1066 (2015).

47. Samir, P. et al. DDX3X acts as a live-or-die checkpoint in stressed cells by regulating NLRP3 inflammasome. Nature 573, 590-594 (2019).

48. Cheson, B. D. et al. Recommendations for initial evaluation, staging, and response assessment of Hodgkin and non-Hodgkin lymphoma: the Lugano classification. J. Clin. Oncol. 32, 3059-3068 (2014).

49. Cheson, B. D. et al. Refinement of the Lugano Classification lymphoma response criteria in the era of immunomodulatory therapy. Blood 128, 2489-2496 (2016).

50. Muhamad, H. et al. Programmed cell death 1 and programmed cell death ligands in extranodal natural killer/T cell lymphoma: expression pattern and potential prognostic relevance. Acta Haematol. 143, 78-88 (2019).

(i) Open Access This article is licensed under a Creative Commons Attribution 4.0 International License, which permits use, sharing, adaptation, distribution and reproduction in any medium or format, as long as you give appropriate credit to the original author(s) and the source, provide a link to the Creative Commons license, and indicate if changes were made. The images or other third party material in this article are included in the article's Creative Commons license, unless indicated otherwise in a credit line to the material. If material is not included in the article's Creative Commons license and your intended use is not permitted by statutory regulation or exceeds the permitted use, you will need to obtain permission directly from the copyright holder. To view a copy of this license, visit http://creativecommons. org/licenses/by/4.0/.

(c) The Author(s) 2020 